\title{
O DESENVOLVIMENTO DE COMPETÊNCIAS EXIGIDAS PELA ENGENHARIA 4.0 DENTRO DE UM MODELO DE EDUCAÇÃO TUTORIAL
}

Eduardo Rodrigues Neto-eduardo.neto@ctec.ufal.br

Universidade Federal de Alagoas, Centro de Tecnologia

Av. Lourival Melo Mota, S/N, Tabuleiro do Martins

57072-900 - Maceió - Alagoas

José Matheus Anacleto Caldeira Alves-jmatheusalves3@gmail.com

Universidade Federal de Alagoas, Centro de Tecnologia

Av. Lourival Melo Mota, S/N, Tabuleiro do Martins

57072-900 - Maceió - Alagoas

Rayanne Lira Barros - rayannelirabarroos@gmail.com

Universidade Federal de Alagoas, Centro de Tecnologia

Av. Lourival Melo Mota, S/N, Tabuleiro do Martins

57072-900 - Maceió - Alagoas

Willian Tcheldon Oliveira Santos - tcheldon.ufal@gmail.com

Universidade Federal de Alagoas, Centro de Tecnologia

Av. Lourival Melo Mota, S/N, Tabuleiro do Martins

57072-900 - Maceió - Alagoas

Karoline Alves de Melo Moraes - melokarol@gmail.com

Universidade Federal de Alagoas, Centro de Tecnologia

Av. Lourival Melo Mota, S/N, Tabuleiro do Martins

57072-900 - Maceió - Alagoas

Resumo: A quarta revolução industrial, chamada de indústria 4.0, transformará a maneira como o profissional se relaciona e atua no mercado. Neste cenário de constante evolução tecnológica e foco maior nas competências sociais dos indivíduos (soft skills), é premente que o profissional esteja sempre pronto a aprender e acompanhar a constante evolução das ferramentas, e no contexto da engenharia não é diferente, com a nova engenharia, a Engenharia 4.0. A partir disso, dentro da necessidade de transformar a formação do novo engenheiro, se apresenta o Programa de Educação Tutorial - PET, programa que objetiva justamente garantir aos alunos do curso oportunidades de vivenciar experiências não presentes em estruturas curriculares convencionais, promovendo uma formação global aos estudantes. Sendo assim, esse trabalho tem o objetivo realizar um diagnóstico das competências desenvolvidas durante a participação no programa, além de apresentar como o método tutorial é capaz de suprir as necessidades que a nova engenharia requer de seus profissionais. A partir de pesquisa com egressos, foi possível observar que boa parte das competências citadas como necessárias nesse novo cenário foram supridas pelas atividades do programa.

Palavras-chave: Educação Tutorial. Competências. Engenharia 4.0. 


\section{INTRODUÇÃO}

A indústria 4.0, tratada como a quarta revolução industrial (WORLD ECONOMIC FORUM, 2016), vem para alterar a maneira como o profissional se comporta no mercado. Neste cenário, o tema vem sendo cada vez mais pesquisado e debatido, seja por empresários, companhias ou mesmo no ambiente universitário, ressaltando a importância de se repensar a formação e atuação dos futuros profissionais.

O termo "Indústria 4.0" começou a ganhar visibilidade pública na Alemanha em 2011 se tornando inclusive parte da iniciativa do governo intitulada "High-Tech Strategy 2020 for Germany" (GERMANY FEDERAL MINISTRY OF EDUCATION AND RESEARCH, 2014), mas, recentemente, passou a ser mais associado ao trabalho de Klaus Schwab, no seu livro "The Fourth Industrial Revolution" (COSTA, 2018). Assim, é identificada uma grande diferença positiva da quarta revolução industrial para as que a precederam: desta vez a próxima grande revolução já foi prevista, possibilitando a adaptação e preparação de todos.

Historicamente, observa-se que, a cada revolução industrial, as competências necessárias aos trabalhadores se modificaram (AIRES; FREIRE; SOUZA, 2016). Com o surgimento da indústria 4.0, espera-se o aumento da eficiência no trabalho entre $10 \%$ e $25 \%$ e o "surgimento de novas atividades e profissões, que demandarão adaptações no padrão de formação de recursos humanos." (CNI, 2016). Neste âmbito, o mercado tem buscado profissionais com uma formação diferenciada, que estejam sempre dispostos a aprender e se adaptarem a uma indústria que sempre se reinventa.

No contexto da engenharia, surge o termo Engenharia 4.0. Espera-se que o novo engenheiro seja um profissional o mais multidisciplinar possível, agregando à sua atuação disciplinas de outras áreas, como a área de humanas. Além disso, espera-se que o profissional coloque seus conhecimentos em prática usando a criatividade e inovação para solucionar problemas organizacionais (AIRES et al., 2017).

Segundo Schaper et al. (2012, apud. Costa, 2018), as principais competências esperadas ao novo profissional da engenharia dentro do contexto da indústria 4.0 estão divididas em quatro eixos:

- Competências profissionais: Competências linguísticas, conhecimento em tecnologias de informação e comunicação, conhecimento de novas tecnologias, capacidade de administração, gestão de risco e detecção de inconformidades, competências empreendedoras e competências analíticas.

- Competências metodológicas: Resolução de problemas complexos, habilidades cognitivas, conhecimentos em novas tecnologias, criatividade, habilidades interdisciplinares, pensamento crítico.

- Competências sociais: competências interpessoais, capacidade de comunicação, capacidade de relacionamento intercultural, colaboração virtual, coordenação com os outros, mente aberta, competências de negociação, gestão de pessoas, ética e responsabilidade social e orientação para o serviço.

- Competências pessoais: gestão de tempo, conhecimento em psicologia e linguagem corporal, lidar com a persistência e pressão, inteligência emocional, julgamento e tomada de decisão e capacidades intra-empreendedoras.

Neste cenário, segundo o Manual de Orientações Básicas - MOB (2006), o Programa de Educação Tutorial - PET tem como objetivo garantir aos alunos oportunidades de vivenciar experiências não presentes em estruturas curriculares convencionais, visando a sua formação global e favorecendo a formação acadêmica, tanto para a integração do mercado profissional quanto para o desenvolvimento em programas de pós-graduação. 
O método tutorial permite o desenvolvimento de habilidades de resolução de problemas e pensamento crítico entre os bolsistas, em contraste com o ensino centrado principalmente na memorização passiva de fatos e informações, e oportuniza aos estudantes tornarem-se cada vez mais independentes em relação à administração de suas necessidades de aprendizagem (MOB, 2006).

A ação em grupo e a dedicação ao curso permitem desenvolver a capacidade de trabalho em equipe, facilitar a compreensão das características e dinâmicas individuais, bem como a percepção da responsabilidade coletiva e do compromisso social (MOB, 2006).

Por conseguinte, o programa tem como objetivos principais: oferecer uma formação acadêmica de excelente nível, visando a formação de um profissional crítico e atuante; promover a integração da formação acadêmica com a futura atividade profissional, especialmente no caso da carreira universitária; estimular a melhoria do ensino de graduação, através da educação tutorial, formando jovens versáteis, de iniciativa, de expressão oral e argumentação.

Dessa forma, o PET Engenharia Civil da Universidade Federal de Alagoas realiza atividades baseadas no MOB e na tríade (ensino, pesquisa e extensão) que garantem o desenvolvimento do perfil do egresso no curso e assegura a formação do engenheiro inovador, empreendedor e professor.

Diante disso, o presente artigo tem como objetivo realizar um diagnóstico das competências desenvolvidas durante a participação no programa pelo membros, além de apresentar como o método tutorial, base do programa e formador de competências globais, é capaz de suprir as necessidades que a nova engenharia requer de seus profissionais, garantindo uma formação global e um profissional mais preparado para as diferentes realidades de desafios encontrados no mercado de trabalho.

\section{METODOLOGIA}

Inicialmente, o trabalho foi baseado em uma revisão de literatura em bases nacionais e internacionais, buscando identificar as competências e habilidades requeridas para o novo profissional da área da engenharia dentro do contexto da indústria 4.0.

De posse de quais seriam essas competências e de modo a diagnosticar quais delas foram desenvolvidas pelos profissionais da Engenharia Civil, formados pela Universidade Federal de Alagoas e que fizeram parte do Programa de Educação Tutorial - PET Engenharia Civil da UFAL, foi elaborado um questionário, no GoogleForms, contendo perguntas que circundam a temática. Dentre as perguntas, foram exploradas as competências profissionais, metodológicas, sociais e pessoais, citadas por Schaper et al. (2012, apud. Costa, 2018).

Ao aplicar o questionário, houve o retorno de 29 profissionais formados pela UFAL e que participaram do PET Engenharia Civil, dos quais, atualmente, 55,2\% apresentam formação de mestre, $20,7 \%$ de doutor, $17,2 \%$ de graduando e 6,9\% de pós-graduando, distribuídos nas diferentes áreas da engenharia (Figura 1). 
Figura 1 - Área de atuação dos profissionais da engenharia que passaram pelo PET Engenharia Civil da UFAL.

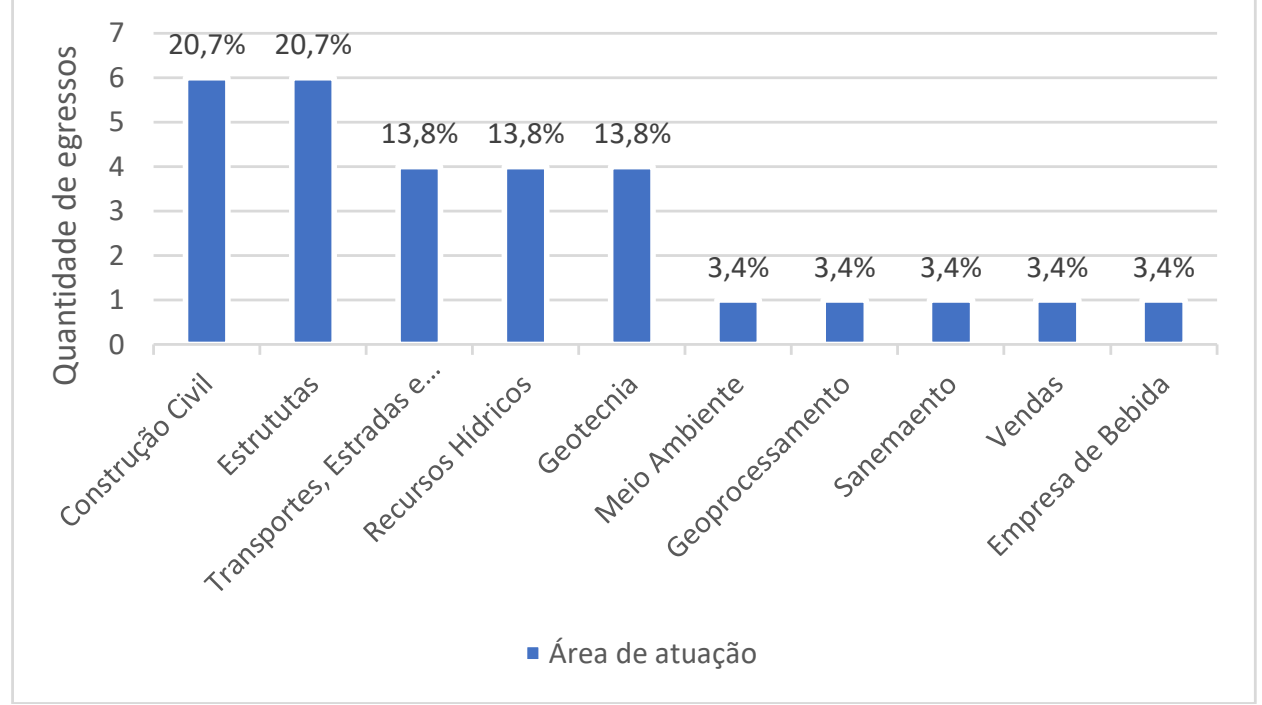

Fonte: Autores (2019)

\section{RESULTADOS E DISCUSSÃO}

O Programa de Educação Tutorial - PET Engenharia Civil da UFAL, a partir da realização de atividades baseadas na tríade: ensino, pesquisa e extensão, tem permitido os alunos integrantes do Programa vivenciarem experiências extracurriculares. A atuação desses alunos nas atividades propostas no planejamento anual no grupo contribuiu para o desenvolvimento de diversas competências profissionais, metodológicas, sociais, pessoais e de auto-competência, as quais não seriam possíveis adquirir somente com a presença nas aulas das disciplinas obrigatórias e eletivas da grade curricular do curso.

Dessa forma, os resultados apresentados a seguir reúnem as impressões de 29 pessoas (profissionais que participaram do PET Engenharia Civil da UFAL durante algum momento da graduação) que puderam responder o questionário aplicado e contribuir para o diagnóstico das competências desenvolvidas durante o período de permanência no Programa.

Ao serem questionados acerca das competências profissionais, a maioria dos entrevistados respondeu que a participação no Programa permitiu desenvolver as competências listadas (Figura 2), dando destaque às ligadas ao domínio da escrita, compreensão textual, organização de informações, além das relacionadas ao empreendedorismo. Das competências profissionais, listadas por Schaper et al. (2012, apud. Costa, 2018), as competências analíticas foram as que menos foram desenvolvidas segundo a análise das respostas dos questionários no eixo das competências profissionais, representando apenas $27,6 \%$ dos entrevistados. 


\section{COBENGE (C) COBENGE 2020 da ABENGE}

\section{"Os desafios para formar hoje o engenheiro do amanhã"}

Figura 2 - Diagnóstico das competências profissionais desenvolvidas pela atuação no PET Engenharia Civil da UFAL.

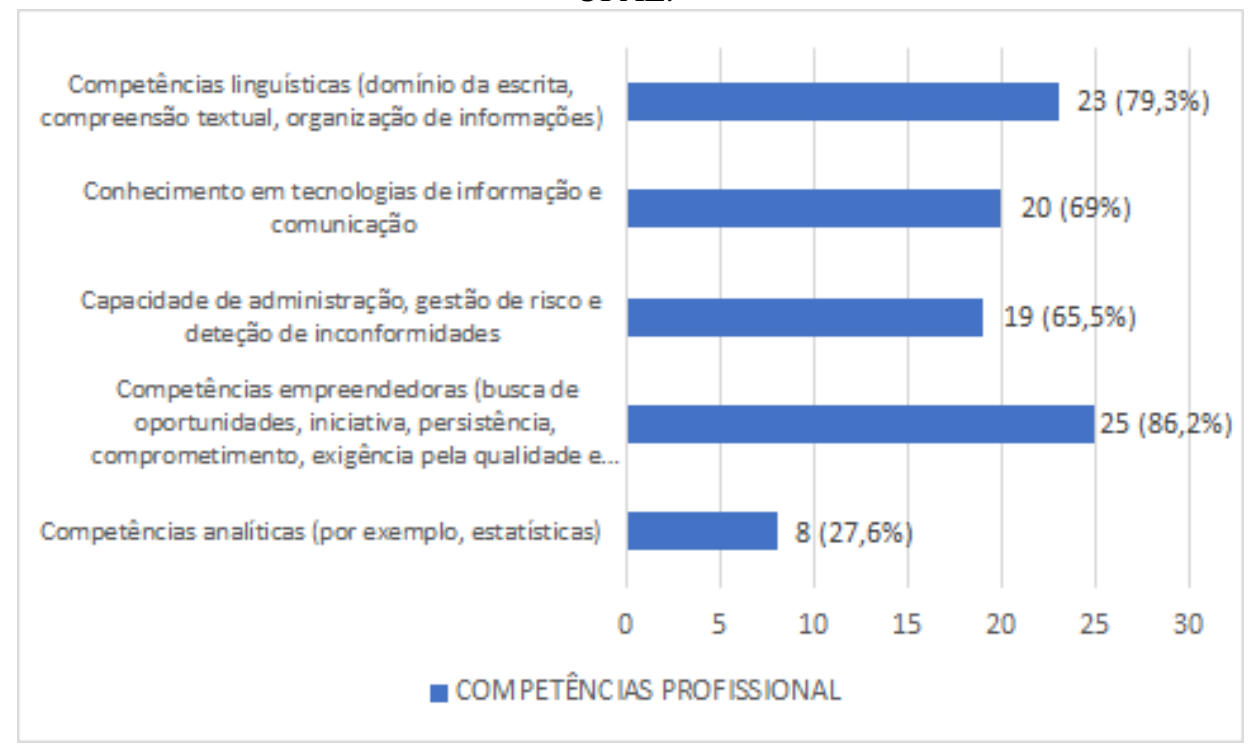

Fonte: Autores (2019)

Relacionando as competências profissionais listadas acima com as atividades do PET Engenharia Civil, constata-se que essas são desenvolvidas por meio de atividades ligadas à pesquisa, elaboração de projetos, sejam eles de cunho acadêmico ou extensionista, e organização de eventos.

O diagnóstico das competências metodológicas (Figura 3) mostraram um forte desenvolvimento de habilidades associadas à resolução de problemas complexos, criatividade, pensamento crítico, além das habilidades interdisciplinares. De modo aproximado, apenas metade dos entrevistados afirmaram o desenvolvimento de habilidades cognitivas e de conhecimento em novas tecnologias.

Figura 3 - Diagnóstico das competências metodológicas desenvolvidas pela atuação no PET Engenharia Civil da UFAL.

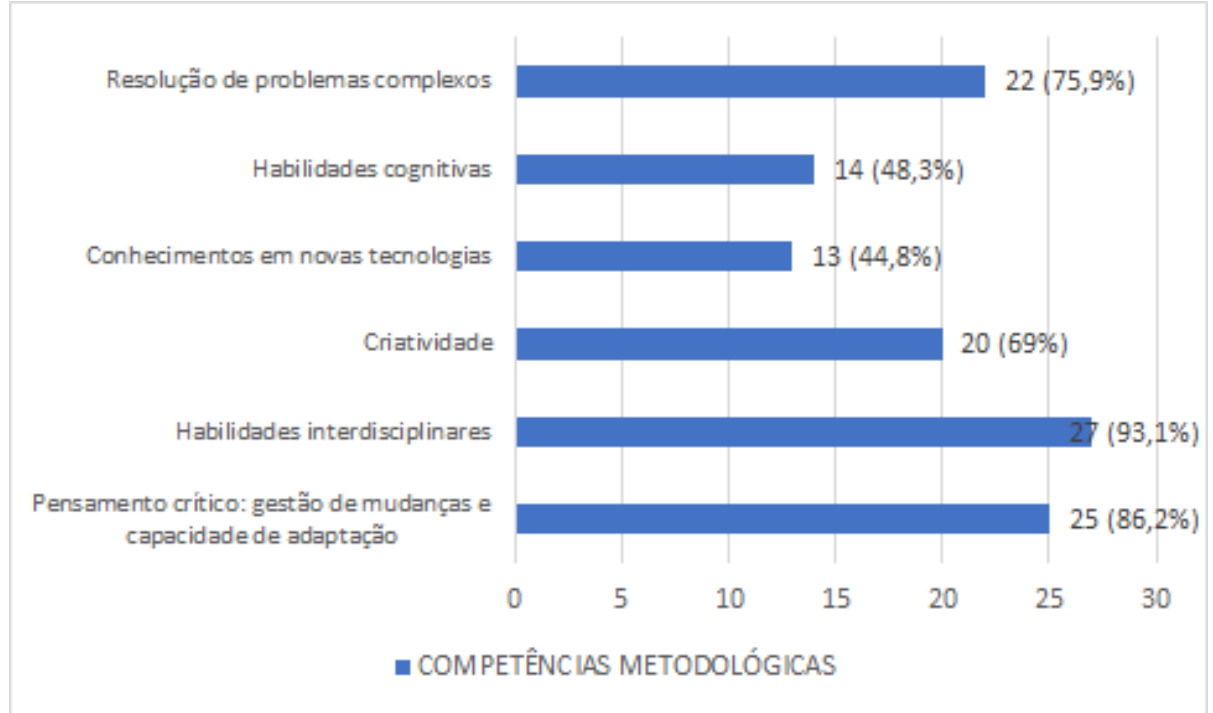

Fonte: Autores (2019) 
A criatividade desenvolvida está bastante relacionada com a capacidade de resolução de problemas, sejam eles provenientes de pesquisas/estudos, como de problemas organizacionais que surgem em ambientes em que há trabalho em grupo. Ser criativo é estar preparado para lidar com as dificuldades que aparecem no dia a dia, buscando as melhores soluções e aproveitando diferentes oportunidades.

As habilidades interdisciplinares são desenvolvidas pelo trabalho em conjunto com outros grupos PET ou outros grupos acadêmicos, presentes na Instituição de Ensino (IES), podendose citar a organização de encontros, seminários, ações de extensão e ações de promoção à saúde mental dos estudantes.

Desenvolver o pensamento crítico é uma das habilidades mais trabalhadas dentro do contexto do PET, e em específico, do PET Engenharia Civil da Ufal, uma vez que os alunos estão constantemente debatendo questões relacionadas à educação e políticas afirmativas, podendo citar atividades como o "Acorda Civil!" e o Aprofunda PET, respectivamente. Nessas atividades, o "Acorda Civil!" teve como objetivo abrir um espaço de discussão entre os discentes e docentes do Centro de Tecnologia acerca das melhorias do curso e discutir sobre as novas metodologias de ensino, já o AprofundaPET teve como objetivo trabalhar temas transversais internamente no grupo PET. Além dessas atividades, o incentivo à participação em eventos educacionais/científicos/PET e a presença em diferentes espaços de discussão com pessoas com pensamento variado, potencializam o desenvolvimento do pensamento crítico e a capacidade de se adaptar diante das necessidades.

Dentro das competências sociais (Figura 4), de modo geral, todas foram exploradas, no entanto, houve destaque ao desenvolvimento das competências interpessoais e das capacidades de comunicação, de relacionamento intercultural, coordenação, gestão de pessoas e ética e responsabilidade.

Figura 4 - Diagnóstico das competências sociais desenvolvidas pela atuação no PET Engenharia Civil da UFAL.

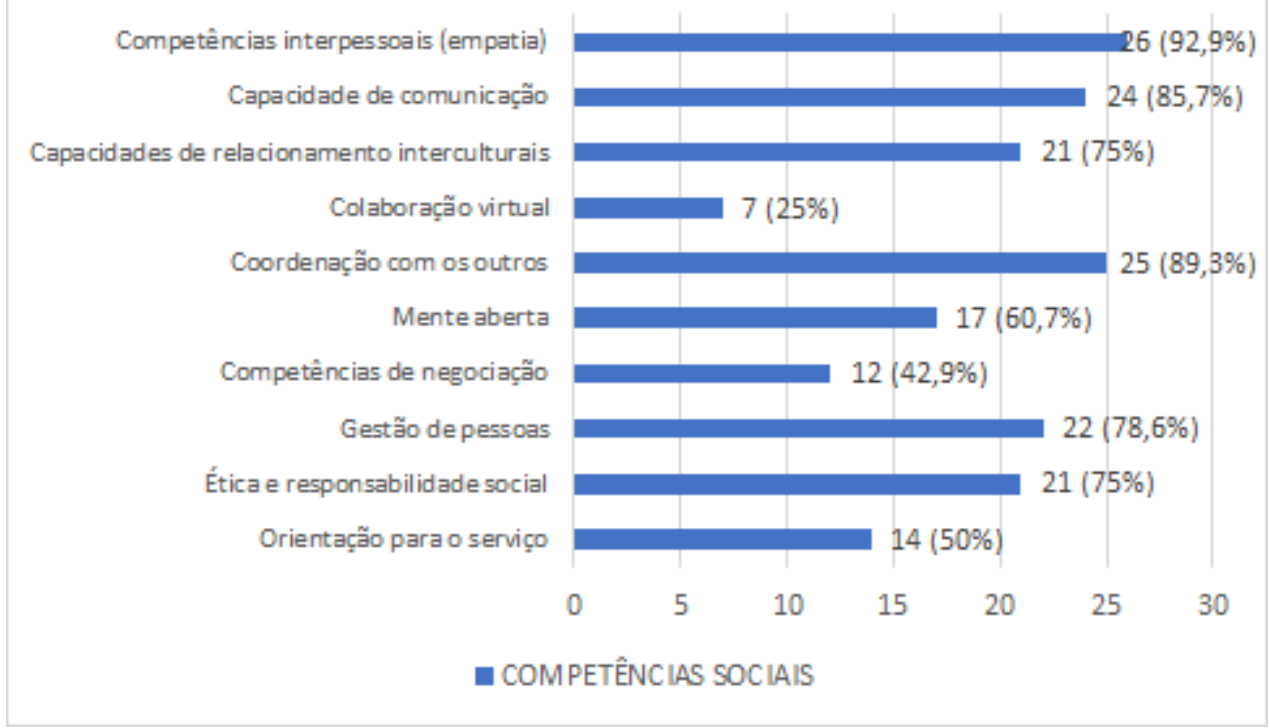

Fonte: Autores (2019)

Por meio de atividades internas do grupo PET Engenharia Civil, como as reuniões semanais, liderança de equipes de trabalho e gestão do grupo, as competências sociais podem ser desenvolvidas, preparando os alunos para ambientes corporativos e profissionais. Vale destacar que as competências de negociação são desenvolvidas durante a realização do maior simpósio técnico-científico de Engenharia Civil do estado de Alagoas, no qual os membros são 
colocados em situações de negociação com fornecedores, palestrantes e empresas para obtenção de patrocínio financeiro para o evento. Dentro de uma única atividade, como o simpósio citado, há o desenvolvimento de outras competências sociais como: comunicação, gestão de pessoas, coordenação, ética e responsabilidade.

Em relação às competências pessoais e auto-competências (Figura 5), as habilidades de gestão de tempo, persistência e pressão, além de julgamento e tomada de decisão, foram as mais exploradas dentro desse eixo de competências.

Figura 5 - Diagnóstico das competências pessoais e auto-competências desenvolvidas pela atuação no PET Engenharia Civil da UFAL.

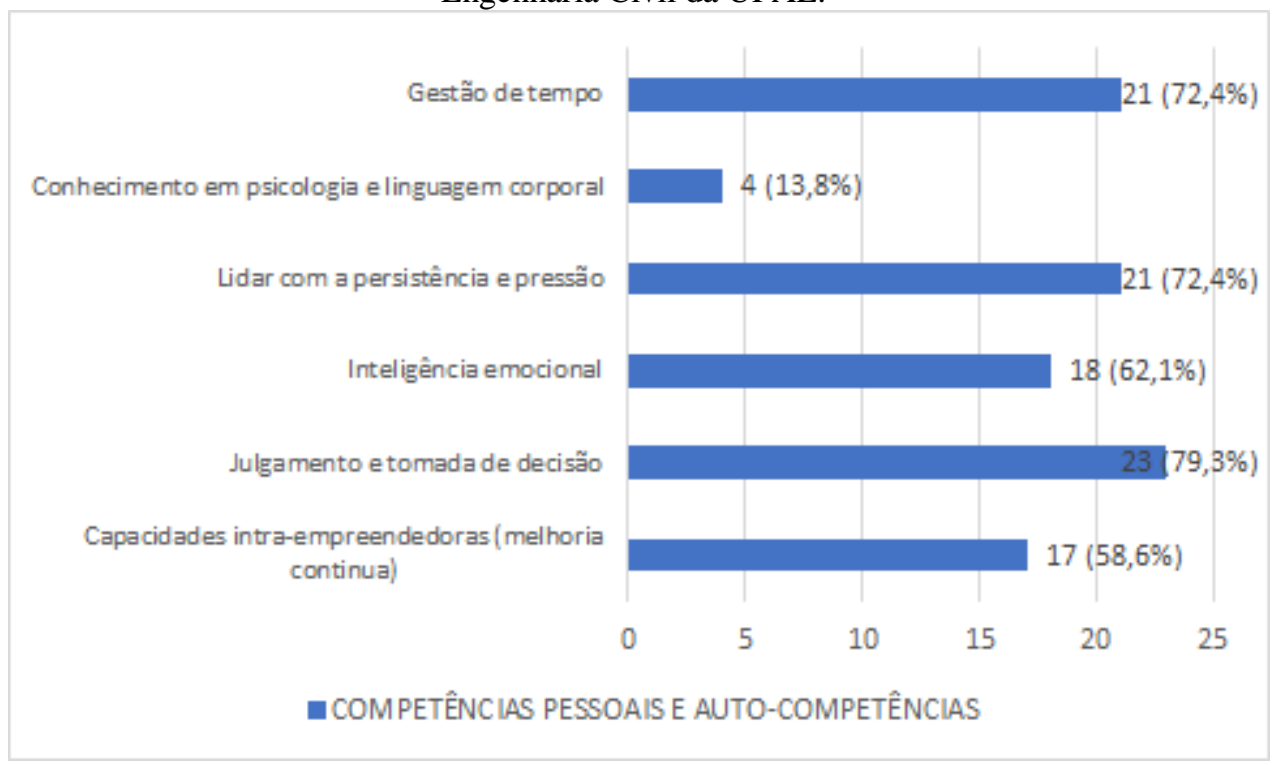

Fonte: Autores (2019)

Estar apto a gerir o próprio tempo é uma capacidade imprescindível para todos os estudantes e profissionais do mercado de trabalho, de modo que todas as atividades, sejam elas pessoais ou profissionais, sejam realizadas dentro do prazo e com qualidade. Além disso, ser persistente é uma característica necessária diante das dificuldades que podem surgir no dia a dia. Ser capaz de julgar uma ação e, consequentemente, definir uma decisão é fundamental quando o profissional se encontra em situação de coordenação e liderança de uma equipe de trabalho.

Além da análise quantitativa do diagnóstico das competências desenvolvidas pelos profissionais que passaram pelo Programa de Educação Tutorial - PET Engenharia Civil da UFAL, os entrevistados foram questionados, através de uma pergunta aberta, em como essas competências têm influenciado na sua atuação como profissional da engenharia. Dessa forma, as principais respostas encontram-se vinculadas à necessidade de saber lidar com diferentes personalidades no ambiente de trabalho, prezando pelas relações interpessoais e à capacidade de comunicação, de tomada de decisão e de resolução de problemas.

Vale destacar que em uma das respostas abertas, um dos entrevistados afirmou que a atuação em Programas como o PET funciona como um "laboratório", em que as pessoas desenvolvem competências e habilidades, permitindo assim, uma preparação para o mercado de trabalho de forma mais completa e diversificada.

Diante disso, pode-se constatar que o Programa de Educação Tutorial - PET Engenharia Civil da UFAL, através de suas atividades, tem contribuído de forma significativa para o desenvolvimento pessoal e profissional dos estudantes, configurando um espaço de suma 
importância no que se refere ao ato de desenvolver competências globais exigidas pelo futuro da engenharia.

\section{CONCLUSÃo}

Considerando as competências requeridas ao profissional da engenharia dentro do contexto da globalização 4.0, pode-se concluir que muitas dessas competências são desenvolvidas através de experiências extracurriculares disponibilizadas pelas atividades realizadas pelo PET Engenharia Civil da UFAL e das ferramentas nele disponibilizadas. Isso, possibilita, intrinsecamente, um maior domínio sobre às demandas exigidas pelo atual mercado, e, portanto, permite um maior discernimento na resolução de problemas mais complexos, associados às auto-competências, estruturação de equipe e habilidades interdisciplinares.

\section{REFERÊNCIAS}

AIRES, R. W. A.; MOREIRA, F. K.; FREIRE, P. S. Indústria 4.0: Competências requeridas aos profissionais da quarta revolução industrial. In: VII Congresso Internacional de Conhecimento e Inovação, 2017, Foz do Iguaçu. Anais Ciki. Foz do Iguaçu: UFSC, 2017.

AIRES, R. W. A.; FREIRE, P. S.; SOUZA, J. A. Educação Corporativa como ferramenta para estimular a inovação nas organizações: uma revisão de literatura. In: CONGRESSO BRASILEIRO DE GESTÃO DO CONHECIMENTO - KM BRASIL, 13., 2016, São Paulo. Anais... São Paulo: SBGC, 2016.

Confederação Nacional da Indústria. Desafios para a indústria 4.0 no Brasil / Confederação Nacional da Indústria. - Brasília: CNI, 2016. 34 p.

COSTA, F.M.P. Identificar e caracterizar as competências necessárias ao profissional de Engenharia e Gestão Industrial para enfrentar a Indústria 4.0. Dissertação de mestrado. Universidade do Minho, 2018.

Germany Federal Ministry of Education and Research. The new High-Tech Strategy Innovations for Germany. Federal Ministry of Education and Research (BMBf), 2014.

MINISTÉRIO DA EDUCAÇÃO - MEC. Programa de Educação Tutorial - PET. Manual de Orientações Básicas - MOB. Brasília, dez. 2006. 


\title{
THE DEVELOPMENT OF COMPETENCES REQUIRED BY ENGINEERING 4.0 WITHIN A MODEL OF TUTORIAL EDUCATION
}

\begin{abstract}
The fourth industrial revolution, called industry 4.0, will transform the way the professional relates to each other and acts in the marketplace. In this scenario of constant technological evolution and greater focus on the social skills of individuals (soft skills), it is imperative that the professional is always ready to learn and follow the constant evolution of tools, and in the context of engineering is no different, with the new engineering, the Engineering 4.0. From this, within the need to transform the formation of the new engineer, is presented the Program of Tutorial Education - PET, a program that aims to guarantee the students of the course opportunities to live experiences not present in conventional curricular structures, promoting a global training to the students. Therefore, this work has the objective to make a diagnosis of the competences developed during the participation in the program, besides presenting how the tutorial method is able to supply the needs that the new engineering requires of its new professionals. Based on research with graduates, it was possible to observe that most of the competencies cited as necessary in this new scenario were covered by the activities of the program.
\end{abstract}

Key-words: Tutorial Education, Competences, Engineering 4.0. 